\title{
RADIAÇÃO GAMA NA CONSERVAÇÃO PÓS-COLHEITA DA NECTARINA (PRUNUS PERSICA VAR. NUCIPERSICA) FRIGOCONSERVADA ${ }^{1}$
}

\author{
LEANDRO CAMARGO NEVES ${ }^{2}$, RODRIGO LILLA MANZIONE³ ${ }^{3}$ ROGÉRIO LOPES VIEITES ${ }^{4}$.
}

\begin{abstract}
RESUMO - O trabalho foi desenvolvido no laboratório de Frutas e Hortaliças do Departamento de Gestão e Tecnologia Agroindustrial da Faculdade de Ciências Agronômicas - UNESP - Câmpus de Botucatu, tendo como objetivo principal a caracterização do comportamento da radiação gama, na conservação pós-colheita da nectarina cv. "Sunred”. Os frutos foram colhidos no início do estádio de maturação, selecionados, limpos, pré-resfriados ( $4^{\circ} \mathrm{C}$ por 12 horas) e submetidos a diferentes doses de radiação gama, constituindo assim os tratamentos: $10,0 \mathrm{kGy}, 2-0,2 \mathrm{kGy}, 3-0,4 \mathrm{kGy}, 4-0,6 \mathrm{kGy}$, $5-0,8 \mathrm{kGy}$, sendo após armazenados em câmara fria com temperaturas de $0^{\circ} \mathrm{C}$ e $90-95 \%$ de UR, por 28 dias. As análises foram realizadas a cada sete dias, determinando-se o aspecto visual dos frutos, a perda de massa fresca, a firmeza de polpa, a acidez total titulável (ATT), os sólidos solúveis totais (STT) e a razão STT/ATT. Após 28 dias de armazenamento, verificou-se que os frutos submetidos à dose de 0,4 kGy apresentaram o melhor aspecto visual, as menores perdas de massa fresca, e a maior firmeza de polpa, não ocorrendo, entretanto, variações significativas nos teores de acidez total titulável, sólidos solúveis totais e nos valores da razão SST/ATT.
\end{abstract}

Termos para indexação: cobalto 60, nectarina cv. "Sunred", radiação ionizante

\section{GAMA RADIATION IN POSTHARVEST CONSERVATION OF NECTARINA (PRUNUS PERSICA VAR. NUCIPERSICA) COOLED STORAGE.}

\begin{abstract}
This work was developed at the Fruit and Vegetable laboratory in the Agro-Industrial Department of the Agriculture College - UNESP - Botucatu campus, having as a main objective the characterization of gamma radiation in nectarine cv. Sunred post harvest storage. The fruits were picked out at the beginning of the maturation stage, selected, cleaned, pre cooled ( $4^{\circ} \mathrm{C}$ for 12 hours) and then submitted to different gama radiation doses, constituting the treatments: $1-0,0 \mathrm{kGy}, 2-0,2 \mathrm{kGy}, 3-0,4 \mathrm{kGy}, 4-0,6 \mathrm{kGy}, 5-0,8 \mathrm{kGy}$. They were then storaged in a cold chamber at $0^{\circ} \mathrm{C}$ and $90-95 \%$ of RH during 28 days. The analysis were performed each seven days determining fruits visual aspect, fresh mass loss, pulp firmness, titratable acidity (TA), total soluble solids (TSS) and the ratio (TSS/TA). After 28 days storage it was verified that the fruits submitted to $0,4 \mathrm{kGy}$ dose presented the best visual aspect, lesser loss of fresh mass and higher pulp firmness. However, it was not found meaningful variation in the contents of titratable acidity, total soluble solids and the values in the ratio.
\end{abstract}

Index Terms: Cobalt 60, Sunred cv. nectarine, ionized irradiation.

\section{INTRODUÇÃO}

A nectarina (Prunus persica var. nucipersica), fruto de origem chinesa, é um tipo de pêssego com epiderme desprovida de pêlos, tamanho pequeno, polpa firme, aroma e sabor bem acentuados (EMBRAPA, 1984). A cultivar "Sunred", também chamada de Rubrosol, foi criada pelo programa de melhoramento da Universidade da Flórida/EUA, em 1964, é altamente produtiva (de 25 a $40 \mathrm{~kg}$ /planta) e bem adaptada às condições da região Sul do Brasil (Raseira \& Medeiros, 1998).

A irradiação de alimentos consiste na exposição de um dado material, de origem vegetal e/ou animal, à radiação ionizante, proveniente tanto de uma máquina de feixes de elétrons como de fontes radioativas. Apenas as fontes de ${ }^{60} \mathrm{CO}$ e ${ }^{137} \mathrm{Cs}$ são consideradas para uso comercial, devido à produção de raios gama de energias adequadas, disponibilidade e custo, sendo que a fonte de ${ }^{60} \mathrm{CO}$ é a que tem maior aceitação por apresentar-se na forma metálica e ser insolúvel em água, proporcionando assim maior segurança ambiental (Ehlermann, 1990; Food Irradiation, 1996).

O’Beirne (1989) descreve que, no processo de irradiação de alimentos, apenas os raios gama entram em contato com o alimento, sem qualquer risco de contaminação radioativa. As doses de radiação são quantificadas em termos de energia absorvida pelo produto irradiado. A dose de 1 Kilogray (kGy) corresponde à absorção de 1 Kilojoule por quilograma de produto irradiado. As doses normalmente aplicadas aos alimentos situam-se entre 0,1 a 7,0 kGy.

O uso da radiação gama como tecnologia de conservação de alimentos está basicamente ligado a três fatores: tipo de alimento a ser irradiado, dose a ser aplicada e tempo de exposição do alimento à fonte irradiadora (Vieites, 1998), assim como a fatores mais intrínsecos: tipo do tecido, porção da célula exposta ao processo, volume nuclear, idade das células irradiadas, conteúdo de água presente nas células e a fatores bióticos, como temperatura, luminosidade e umidade relativa do ar
(Grossman \& Craig, 1982) antes e, principalmente, durante e depois do processo de irradiação.

A irradiação gama pode estender a vida de prateleira de muitos frutos perecíveis, pelo controle da deterioração causada por microorganismos, atraso do amadurecimento e da senescência propriamente dita (Urbain, 1986). Damayanti et al. (1992), prolongaram o período de conservação pós-colheita, em abacaxis cv. 'Queen', pelo controle de fungos causadores de podridões, utilizando doses de radiação gama entre 0,05 e 0,25 kGy. Germano et al. (1996), trabalhando com abacates da variedade 'Fortuna', conseguiram, através da aplicação de radiação gama, um incremento no armazenamento refrigerado de quatro dias para dose de $0,08 \mathrm{kGy}$ e de oito dias para dose de $0,1 \mathrm{kGy}$, que inicialmente era de sete dias. Cia et al. (2000) recomendam doses de radiações gama entre 0,5 e 2 kGy, no controle de Botritis cinerea, em uva 'Itália'.

Segundo United Fresh Fruit - Vegetable Association (1986), para utilizar-se radiações ionizantes na desinfecção de alimentos e no aumento da conservação pós-colheita de frutas e hortaliças, alguns critérios devem ser levados, em conta, a saber: o alimento precisa ter tolerância mais elevada do que o inseto ou o microorganismo; o tratamento requerido deve ser tão ou mais econômico que outros tratamentos efetivos; o tratamento deve ser compatível com os aspectos legais estabelecidos pelas autoridades sanitárias, isto é, deve ser inócuo à saúde do consumidor; e, sobretudo, obedecer à legislação vigente do país importador.

A FAO (1993) estima que $25 \%$ de toda a produção mundial é perdida pela ação de insetos, bactérias e roedores. O uso da técnica de irradiação para a conservação de alimentos, por si só, não soluciona todos os problemas de perdas, pois, ao contrário dos métodos químicos convencionais, a irradiação não possui efeito residual, devendo-se preservar em condições assépticas o alimento após ser irradiado, evitando assim uma nova reinfestação (Gciia, 1991).

Os custos líquidos da irradiação oscilam entre 10 e 15 dólares americanos por tonelada, no caso da aplicação de uma dose baixa (até 1

1 (Trabalho 179/2001). Recebido: 13/11/2001. Aceito para publicação: 06/09/2002.

2 UFRGS - Eng. Agr. Msc. Doutorando em Fitotecnia, Cx. Postal 776, CEP 91501-970, Porto Alegre/RS. e-mail: rappelrs@uol.com.br

3 UNESP/FCA - Eng. Agr., Mestrando em Energia na Agricultura, Cx. Postal 237, CEP 18603-970 Botucatu/SP.

4 UNESP/FCA - Professor Dr. do Depto de Gestão e Tecnologia Agroindustrial, Cx. Postal 237, CEP 18603-970 Botucatu/SP. 
kGy), como, por exemplo, para inibir a germinação em batatas e cebolas ou retardar o amadurecimento de frutos. Variam entre 100 e 250 dólares americanos por tonelada, no caso de aplicação de uma dose alta (acima de 10 kGy), como, por exemplo, para garantir a qualidade higiênica de algumas especiarias (Morrisson \& Roberts, 1990). Estes custos são competitivos com os de outros tratamentos convencionais, e, em alguns casos, a irradiação pode ser mais barata, dependendo do tipo de produto, quantidade e distância do campo de produção até a fonte irradiadora (Gciia, 1991).

Portanto, o presente trabalho visa a um prolongamento na vida útil de prateleira da nectarina cv. "Sunred", através da aplicação de radiação gama em diferentes dosagens.

\section{MATERIAL EMÉTODOS}

O experimento foi conduzido no laboratório de Frutas e Hortaliças, do Departamento de Gestão e Tecnologia Agroindustrial, da Universidade Estadual Paulista - UNESP - Câmpus de Botucatu. Os frutos colhidos no início do estádio de maturação (coloração verdeavermelhada), em propriedade rural no município de Botucatu-SP, foram selecionados, limpos, pré-resfriados a $4^{\circ} \mathrm{C}$ por 12 horas, sendo então encaminhados ao Centro de Energia Nuclear na Agricultura-ESALQ/ USP, localizado em Piracicaba-SP, onde as nectarinas receberam a aplicação de raios gama, que têm como fonte $0^{60} \mathrm{CO}$. O irradiador utilizado foi o "GAMMABEAN 650", e para a constituição dos tratamentos, as doses foram as seguintes: $1-0,0 \mathrm{kGy} ; 2-0,2 \mathrm{kGy} ; 3-0,4 \mathrm{kGy} ; 4-0,6 \mathrm{kGy}$; $5-0,8 \mathrm{kGy}$. A dose aplicada em cada tratamento foi obtida em função da variação do tempo de exposição do fruto à fonte irradiadora. Após aplicação das doses pre-determinadas, as nectarinas foram colocadas em câmara fria com $0^{\circ} \mathrm{Ce}$ 90-95\% de UR, segundo recomendação de Chitarra \& Chitarra (1990). As análises foram realizadas a cada sete dias, 24 horas após a retirada de cada amostra da câmara fria (simulação de comercialização), por um período de 28 dias, sob os seguintes aspectos:

$\mathrm{O}$ aspecto visual do fruto foi avaliado, através de uma escala subjetiva de valores definida pelos autores, com base no avanço do amadurecimento bem como na ocorrência de injúrias e podridões que podem comprometer a qualidade comercial do produto, sendo: $0,4-$ ótimo; 0,3-bom; 0,2-regular; 0,1-ruim.

A perda de massa fresca foi obtida pela pesagem direta dos frutos em balança “OWA LABOR", com resultados expressos em porcentagem.

A firmeza de polpa foi avaliada através do penetrômetro - Fruit pressure test mod.FT327 (3-27 lbs), ponteira de $8 \mathrm{~mm}$.

Os sólidos solúveis totais foram avaliados através da leitura refratométrica direta, em graus Brix, com o refratômetro tipo Abbe, marca ATAGO-N1.

O conteúdo de acidez total titulável, expresso em Cmol.L ${ }^{-1}$, foi obtido através da titulação de $10 \mathrm{~g}$ de polpa, homogeneizada e diluída para $100 \mathrm{~mL}$ de água destilada, com solução padronizada de hidróxido de sódio a $0,1 \mathrm{~N}$.

A razão entre os valores de sólidos solúveis totais e acidez total titulável foi obtida através da fórmula SST/ATT.

$\mathrm{O}$ delineamento experimental empregado no experimento foi o inteiramente casualizado, com esquema fatorial $5 \times 5$ (doses de radiação e épocas de amostragem), com 5 tratamentos e 3 repetições. Os dados foram submetidos à análise de variância e à comparação de médias pelo teste DMS $(\mathrm{P} \leq 0,05)$.

\section{RESULTADOSE DISCUSSÃO}

A Tabela I mostra-nos de forma significativa que os frutos pertencentes ao tratamento 3 , dose de $0,4 \mathrm{kGy}$, apresentaram ao final do período experimental uma melhor manutenção da qualidade visual, não observando, nos frutos submetidos a essa dose, nenhuma ocorrência de deteriorações ou podridões. É importante ressaltar que a queda da qualidade do aspecto visual, detectada nos frutos submetidos a doses superiores a $0,4 \mathrm{kGy}$, não foi de origem microbiológica; isto pode ser explicado pelo fato de que a radiação gama, em doses acima de um limiar, pode interferir nos processos fisiológicos, acelerando o metabolismo e acarretando amadurecimento precoce dos frutos. Estes resultados são semelhantes aos obtidos por Thomas et al. (1995) no qual destaca a eficiência da radiação gama, aplicada em uvas, quanto a uma redução da incidência de podridões em pós-colheita e por Urbain (1986), em abacaxis cv. "Queen," e Zuleta (1989), em mangas cv. "Tommy Atkins”, nos quais afirmam que a radiação gama pode promover o aumento da sua vida útil pelo controle de deteriorações causadas por microorganismos e pelo atraso do amadurecimento e da senescência.

TABELA 1- Evolução do aspecto visual, através de uma escala subjetiva de valores, em nectarinas cv. "Sunred", sob aplicação de radiação gama, ao longo de vinte e oito dias de frigoconservação.

\begin{tabular}{lccccc}
\hline DOSES & & DIAS & DE & CONSERVACC̃̃O & \\
\hline & $\mathbf{0}$ & $\mathbf{7}$ & $\mathbf{1 4}$ & $\mathbf{2 1}$ & $\mathbf{2 8}$ \\
\hline 0,0 kGy & $0,40 \mathrm{a}$ & $0,33 \mathrm{a}$ & $0,27 \mathrm{a}$ & $0,20 \mathrm{c}$ & $0,17 \mathrm{bc}$ \\
$\mathbf{0 , 2}$ kGy & $0,40 \mathrm{a}$ & $0,37 \mathrm{a}$ & $0,33 \mathrm{a}$ & $0,30 \mathrm{~b}$ & $0,23 \mathrm{~b}$ \\
0,4 kGy & $0,40 \mathrm{a}$ & $0,40 \mathrm{a}$ & $0,40 \mathrm{a}$ & $0,40^{\mathrm{a}}$ & $0,40 \mathrm{a}$ \\
0,6 kGy & $0,40 \mathrm{a}$ & $0,30 \mathrm{a}$ & $0,27 \mathrm{a}$ & $0,20 \mathrm{c}$ & $0,20 \mathrm{~b}$ \\
0,8 kGy & $0,40 \mathrm{a}$ & $0,27 \mathrm{a}$ & $0,27 \mathrm{a}$ & $0,17 \mathrm{c}$ & $0,10 \mathrm{c}$ \\
\hline C.V. & 0 & 18,97 & 16,84 & 10,19 & 16,60 \\
D.M.S. & 0 & 0,1698 & 0,1386 & 0,0693 & 0,0980 \\
\hline
\end{tabular}

Médias seguidas da mesma letra, nas colunas, não diferem entre si, significativamente, ao nível de $5 \%$ de probabilidade, pelo teste de Tukey.

Pelos dados obtidos na Tabela II, observou-se que as nectarinas irradiadas a uma dose de $0,4 \mathrm{kGy}$ apresentaram, ao longo do período de frigoconservação, um melhor controle da perda de massa fresca dos frutos. Os frutos da testemunha e os frutos irradiados com a dose de 0,8 $\mathrm{kGy}$ foram os que mais perderam em porcentagem de peso, como os frutos da testemunha devido aos processos normais referentes ao amadurecimento; já os frutos submetidos a doses acima de $0,8 \mathrm{kGy}$, provavelmente devido ao fato de que a dose elevada de radiação pode ter aumentado a intensidade respiratória, proporcionaram maiores perdas de água. Estes dados são concordantes com Dennison \& Ahmed (1975) e Murray et al. (1981) que, trabalhando com tomates, recomendam a utilização da radiação gama, em pós-colheita, com a finalidade de diminuição da velocidade dos processos metabólicos envolvidos no amadurecimento, como a respiração e, conseqüentemente, a perda de água. O mesmo é relatado no artigo de O’Beirne (1989) que verificou que a irradiação tem como uma de suas principais funções biológicas o retardo dos processos fisiológicos envolvidos no desenvolvimento dos frutos, acarretando assim um maior tempo de conservação em pós-colheita.

TABELA 2 - Evolução da perda de massa fresca (\%), em nectarinas cv. "Sunred", sob aplicação de radiação gama, ao longo de vinte e oito dias de frigoconservação.

\begin{tabular}{ccccc}
\hline DOSES & & DIAS & DE & CONSERVAÇÃO \\
\hline 0,0 kGy & $15,31 \mathrm{a}$ & $30,60 \mathrm{a}$ & $47,79 \mathrm{a}$ & $\mathbf{2 8}$ \\
$\mathbf{0 , 2} \mathbf{~ k G y}$ & $9,40 \mathrm{bc}$ & $20,90 \mathrm{bc}$ & $33,45 \mathrm{~b}$ & $45,33 \mathrm{a}$ \\
0,4 kGy & $7,43 \mathrm{c}$ & $15,44 \mathrm{c}$ & $23,82 \mathrm{c}$ & $30,98 \mathrm{c}$ \\
0,6 kGy & $10,31 \mathrm{bc}$ & $22,07 \mathrm{~b}$ & $34,70 \mathrm{~b}$ & $49,07 \mathrm{~b}$ \\
$\mathbf{0 , 8}$ kGy & $12,29 \mathrm{ab}$ & $28,38 \mathrm{a}$ & $48,47 \mathrm{a}$ & $74,17 \mathrm{a}$ \\
\hline C.V. & 16,91 & 13,18 & 10,64 & 10,31 \\
D.M.S. & 3,5017 & 5,8559 & 7,5758 & 10,3199 \\
\hline
\end{tabular}

Médias seguidas da mesma letra, nas colunas, não diferem entre si, significativamente, ao nível de $5 \%$ de probabilidade, pelo teste de Tukey.

Com base nos dados obtidos na Tabela III (Firmeza de polpa), pode-se observar que as nectarinas submetidas à dose de $0,4 \mathrm{kGy}$ de radiação gama apresentaram maior firmeza de polpa em todo o período experimental, demonstrando assim a eficiência da dose de radiação gama corretamente aplicada, diminuindo diretamente as perdas de firmeza de polpa. O mesmo é afirmado por United Fresh - Vegetable Association (1986), Gciia (1991), Vieites (1998) nos quais é descrita a eficiência da aplicação da radiação gama, na conservação dos atributos de qualidade, 
na pós-colheita de frutos e hortaliças, em função da dose, do tempo de exposição e do tipo de alimento a ser irradiado, e por Miller \& McDonald (1999), em trabalhos com mamão cv. "Surise Solo", no qual mencionam a interação positiva entre dose de radiação gama $\mathrm{X}$ temperatura de frigoconservação, proporcionando assim uma maior firmeza de polpa.

TABELA 3 - Evolução da firmeza de polpa (g/f), através de penetrômetro manual (8mm), em nectarinas cv. "Sunred", sob aplicação de radiação gama, ao longo de vinte e oito dias de frigoconservação.

\begin{tabular}{cccccc}
\hline DOSES & & DIAS & DE & CONSERVAÇ̃̃ & \\
\hline & $\mathbf{0}$ & $\mathbf{7}$ & $\mathbf{1 4}$ & $\mathbf{2 1}$ & $\mathbf{2 8}$ \\
\hline $\mathbf{0 , 0} \mathbf{k G y}$ & $8,17 \mathrm{a}$ & $7,13 \mathrm{~b}$ & $6,27 \mathrm{c}$ & $5,27 \mathrm{c}$ & $4,07 \mathrm{c}$ \\
$\mathbf{0 , 2} \mathbf{~ k G y}$ & $8,17 \mathrm{a}$ & $7,33 \mathrm{~b}$ & $6,87 \mathrm{~b}$ & $6,07 \mathrm{~b}$ & $5,20 \mathrm{~b}$ \\
$\mathbf{0 , 4} \mathbf{~ k G y}$ & $8,17 \mathrm{a}$ & $7,80 \mathrm{a}$ & $7,40 \mathrm{a}$ & $6,87 \mathrm{a}$ & $6,50 \mathrm{a}$ \\
$\mathbf{0 , 6} \mathbf{~ k G y}$ & $8,17 \mathrm{a}$ & $7,27 \mathrm{~b}$ & $6,80 \mathrm{~b}$ & $6,13 \mathrm{~b}$ & $5,47 \mathrm{~b}$ \\
$\mathbf{0 , 8} \mathbf{~ k G y}$ & $8,17 \mathrm{a}$ & $7,27 \mathrm{~b}$ & $6,07 \mathrm{c}$ & $5,00 \mathrm{c}$ & $3,73 \mathrm{~d}$ \\
\hline C.V. & 0,71 & 1,22 & 1,34 & 2,33 & 2,00 \\
D.M.S. & 0,1550 & 0,2401 & 0,2210 & 0,3668 & 0,2685 \\
\hline
\end{tabular}

Médias seguidas da mesma letra, nas colunas, não diferem entre si, significativamente, ao nível de 5\% de probabilidade, pelo teste de Tukey.

De acordo com os resultados expressos nas Tabelas IV, V e VI referentes à análise de sólidos solúveis totais (SST), acidez total titulável (ATT) e a razão entre SST/ATT, constata-se que, pela não-interferência da radiação gama nos parâmetros nutricionais dos alimentos, relatada em diversos artigos, ao final do período de 28 dias de experimento, os tratamentos propostos não apresentavam diferença significativa entre si, pelo teste de Tukey, ao nível de $5 \%$, fato este comprovado pelo relato de Bande (1990), no qual menciona que frutos e hortaliças devidamente irradiados não apresentam nenhuma toxidez e praticamente mantêm o mesmo valor nutritivo que os processados por outros métodos. $\mathrm{O}$ mesmo ocorreu no artigo de Cia et al. (2000), em experimento com uva Itália, no qual é observado o não-comprometimento nos teores de sólidos solúveis totais e acidez total titulável pela aplicação de radiação gama, e com Zhao et al. (1996) trabalhando com mamão e Domarco et al. (1996) trabalhando com uva Itália, no qual relatam que a radiação gama não exerce efeito significativo nos teores de sólidos solúveis.

TABELA 4 - Evolução de sólidos solúveis totais ( ${ }^{\circ}$ Brix), em nectarinas cv. "Sunred", sob aplicação de radiação gama, ao longo de vinte e oito dias de frigoconservação.

\begin{tabular}{cccccc}
\hline DOSES & & DIAS & DE & CONSERVAÇ̃̃ & \\
\hline & $\mathbf{0}$ & $\mathbf{7}$ & $\mathbf{1 4}$ & $\mathbf{2 1}$ & $\mathbf{2 8}$ \\
\hline $\mathbf{0 , 0} \mathbf{~ k G y}$ & $8,03 \mathrm{a}$ & $8,53 \mathrm{a}$ & $9,47 \mathrm{a}$ & $10,60 \mathrm{a}$ & $11,77 \mathrm{a}$ \\
$\mathbf{0 , 2} \mathbf{~ k G y}$ & $8,03 \mathrm{a}$ & $8,57 \mathrm{a}$ & $9,55 \mathrm{a}$ & $10,30 \mathrm{~b}$ & $11,80 \mathrm{a}$ \\
$\mathbf{0 , 4} \mathbf{~ k G y}$ & $8,03 \mathrm{a}$ & $8,57 \mathrm{a}$ & $9,33 \mathrm{a}$ & $10,10 \mathrm{~b}$ & $11,73 \mathrm{a}$ \\
$\mathbf{0 , 6} \mathbf{~ k G y}$ & $8,03 \mathrm{a}$ & $8,63 \mathrm{a}$ & $9,53 \mathrm{a}$ & $10,13 \mathrm{~b}$ & $11,87 \mathrm{a}$ \\
$\mathbf{0 , 8} \mathbf{~ k G y}$ & $8,03 \mathrm{a}$ & $8,80 \mathrm{a}$ & $9,93 \mathrm{a}$ & $10,77 \mathrm{a}$ & $11,87 \mathrm{a}$ \\
\hline C.V. & 0,72 & 1,96 & 0,89 & 1,03 & 0,44 \\
D.M.S. & 0,1550 & 0,4545 & 0,2273 & 0,2858 & 0,1386 \\
\hline
\end{tabular}

Médias seguidas da mesma letra, nas colunas, não diferem entre si, significativamente, ao nível de 5\% de probabilidade, pelo teste de Tukey.

TABELA 5 - Evolução da acidez total titulável $\left(\mathrm{cmol} . \mathrm{L}^{-1}\right)$, em nectarinas cv. "Sunred", sob da aplicação de radiação gama, ao longo de vinte oito dias de frigoconservação.

\begin{tabular}{lccccc}
\hline DOSES & & DIAS & DE & CONSERVAČ̃̃ & \\
\hline & $\mathbf{0}$ & $\mathbf{7}$ & $\mathbf{1 4}$ & $\mathbf{2 1}$ & $\mathbf{2 8}$ \\
\hline $\mathbf{0 , 0} \mathbf{~ k G y}$ & $1,300 \mathrm{a}$ & $1,053 \mathrm{a}$ & $0,983 \mathrm{ab}$ & $0,901 \mathrm{a}$ & $0,772 \mathrm{a}$ \\
$\mathbf{0 , 2} \mathbf{~ k G y}$ & $1,300 \mathrm{a}$ & $1,055 \mathrm{a}$ & $1,014 \mathrm{a}$ & $0,917 \mathrm{a}$ & $0,781 \mathrm{a}$ \\
$\mathbf{0 , 4} \mathbf{~ k G y}$ & $1,300 \mathrm{a}$ & $1,068 \mathrm{a}$ & $1,014 \mathrm{a}$ & $0,922 \mathrm{a}$ & $0,784 \mathrm{a}$ \\
$\mathbf{0 , 6} \mathbf{~ G G y}$ & $1,300 \mathrm{a}$ & $1,070 \mathrm{a}$ & $1,005 \mathrm{a}$ & $0,913 \mathrm{a}$ & $0,766 \mathrm{a}$ \\
$\mathbf{0 , 8} \mathbf{~ k G y}$ & $1,300 \mathrm{a}$ & $1,047 \mathrm{a}$ & $0,948 \mathrm{ab}$ & $0,897 \mathrm{a}$ & $0,765 \mathrm{a}$ \\
\hline C.V. & 0 & 0,90 & 1,44 & 1,49 & 1,01 \\
D.M.S. & 0 & 0,0255 & 0,0385 & 0,364 & 0,0209 \\
\hline
\end{tabular}

Médias seguidas da mesma letra, nas colunas, não diferem entre si, significativamente, ao nível de 5\% de probabilidade, pelo teste de Tukey.
TABELA 6 - Evolução dos valores da relação SST/ATT, em nectarinas cv. "Sunred", sob aplicação de radiação gama, ao longo de vinte e oito dias de frigoconservação.

\begin{tabular}{lccccc}
\hline DOSES & & DIAS & DE & CONSERVAÇÃO & \\
\hline & $\mathbf{0}$ & $\mathbf{7}$ & $\mathbf{1 4}$ & $\mathbf{2 1}$ & $\mathbf{2 8}$ \\
\hline $\mathbf{0 , 0}$ kGy & $6,1797 \mathrm{a}$ & $8,1077 \mathrm{a}$ & $9,6340 \mathrm{a}$ & $11,7627 \mathrm{a}$ & $15,3483 \mathrm{a}$ \\
$\mathbf{0 , 2} \mathbf{~ k G y}$ & $6,1797 \mathrm{a}$ & $8,1213 \mathrm{a}$ & $9,4247 \mathrm{ab}$ & $11,6680 \mathrm{a}$ & $15,3823 \mathrm{a}$ \\
$\mathbf{0 , 4} \mathbf{~ k G y}$ & $6,1797 \mathrm{a}$ & $8,4027 \mathrm{a}$ & $9,4380 \mathrm{ab}$ & $11,7897 \mathrm{a}$ & $15,1040 \mathrm{a}$ \\
$\mathbf{0 , 6} \mathbf{k G y}$ & $6,1797 \mathrm{a}$ & $8,0660 \mathrm{a}$ & $9,4890 \mathrm{ab}$ & $11,7153 \mathrm{a}$ & $15,4257 \mathrm{a}$ \\
$\mathbf{0 , 8}$ kGy & $6,1797 \mathrm{a}$ & $8,0253 \mathrm{a}$ & $9,2513 \mathrm{~b}$ & $11,7020 \mathrm{a}$ & $15,3527 \mathrm{a}$ \\
\hline C.V. & 0,72 & 2,50 & 0,97 & 0,66 & 2,34 \\
D.M.S. & 0,1193 & 0,5457 & 02461 & 0,2092 & 0,9642 \\
\hline
\end{tabular}

Médias seguidas da mesma letra, nas colunas, não diferem entre si, significativamente, ao nível de 5\% de probabilidade, pelo teste de Tukey.

\section{CONCLUSÕES}

Com base nos objetivos de prolongamento da vida útil de prateleira e caracterização dos parâmetros (físicos e nutricionais) de qualidade durante a frigoconservação da nectarina cv. "Sunred", conclui-se que o uso da radiação gama, na dose de $0,4 \mathrm{kGy}$, é útil quanto a uma melhor conservação dos atributos de qualidade da nectarina cv."Sunred", prolongando seu período de conservação pós-colheita sem influência sobre os parâmetros nutricionais avaliados.

\section{REFERÊNCIASBIBLIOGRÁFICAS}

BANDE, A.L. Aplicaciones de la irradiación de los alimentos: diversos aspectos relacionados. Alimentaria, Madrid, v.27, p.23-28, 1990.

CIA, P.; BENATO, E.A.; ANJOS, V.D.A.; VIEITES, R.L. Efeito da irradiação na conservação de uva 'Itália'. Revista Brasileira de Fruticultura, Jaboticabal, v.22, n. esp. p.62-67, 2000.

CHITARRA, M.I.F.; CHITARRA, A. B. Pós-colheita de frutos e hortaliças. Lavras: ESAL/FAEPE, 1990. 320p.

DAMAYANTI, M.; SHARMA, G.J., KUNDU, S. C. Gamma radiation influences postharvest disease incidence of pineapple fruits. HortScience, Alexandria, v.27, n.7, p.807-808, 1992.

DENNISON, R.A.; AHMED, E.M. Irradiation treatment of fruits and vegetables. In: HAARD, N.F.:SALUNKHE. D.K. Symposium: Postharvest biology and handling of fruits and vegetables. Westport: Conn. Avi., 1975. p.118-129.

DOMARCO, R.E.; SPOTO, M.H.F.; BLUMER, L.; WALDER, J.M.M. Efeito sinérgico da dose de irradiação e aquecimento na vida de prateleira de uva cultivar Itália. In: CONGRESO LATINOAMERICANO, 8., Y Nacional de horticultura, 6., 1996, Montevideo. Resumenes...Montevideo: Color/Suh., 1996. p. 124.

EHLERMANN, D.A.E. Food irradiation. In: SPIESS, W.E.L.; SCHUBERT, $H$. Engineering and food: preservation processes and related techniques. London: Elsevier Applies Science, 1990. v.2, p.760-773.

EMBRAPA. Centro Nacional de Pesquisa de Fruteiras de Clima Temperado (Pelotas, RS). A cultura do Pessegueiro. Pelotas, 1984. 156p.

FAO. Food and Agricultural Organization. Production Yearbook, Roma, 1993, v.47, 254p.

FOOD IRRADIATION A Guidebook: agricultural service division. 2 ed., Rome Italy: FAO, Technomic Publishing 1996. 232p.

GERNANO, R.M.de A.; ARTHUR, V.; WIENDL, F.M. Conservação póscolheita de abacates Pérsia americana MILL., variedade Fortuna e Quintal, por irradiação. Scientia Agrícola, Piracicaba, v. 2/3, n. 53, p.249-53, 1996.

GROSSMANN, H. H.; CRAIG, R. The effect of gamma irradiation of seeds on germination and plant morphology of Pelargonium $\mathrm{X}$ Hortorum L. H. Bailey. Journal of American Society Horticultural Sciences, Alexandria, v. 1, n.107, p.72-75, 1982.

GRUPOCONSULTIVO INTERNACIONAL SOBREIRRADIAÇÃODE ALIMENTOS. A irradiação de alimentos : ficção ou realidade. GCIIA/FAO/AIEA, 1991.38p. 
MILLER, W.R., McDONALD, R. E. Irradiation, stage of maturity at harvest, and storage temperature during ripening affect papaya fruit quality. HortScience, Alexandria, v.34, n.6, p.1112-1115, 1999.

MORRISSON, R.M.; ROBERTS, T. Cost variables for food irradiators in developing countries: food irradiation for developing countries in Africa. IAEA - TECDOC, 1990.576p.

MURRAY, A.J.; BID, C.R.; SCHUCH, W.W.; HOBSON, G.E. Nutritional aspects of food irradiation. Food Irradiation Publisher International: Projects in the field of the irradiation, n.11, p.21-32, 1981.

O'BEIRNE, D. Irradiation of fruits and vegetables: applications and issues. Professional Horticulture, Oxford, v.3, p.12-19, 1989.

RASEIRA, M. do C. B.; NELSON, H. Cultivares: descrição e recomendação. In: RASEIRA, M. do C. B.; MEDEIROS, C. A. B. A cultura do pessegueiro. Brasília : EMBRAPA - SPI; Pelotas - CPACT, 1988. 350p.; il.

THOMAS, P,; BHUSHAN, B.; JOSHI, M.R. Comparasion of the effect of gamma irradiation, heat-treatment combination and sulphur dioxide generating pads on decy and quality of grapes. Journal of food Science and Techonology, Mysore, v.32, n.6, p.477-81, 1995.

UNITED FRESH FRUIT - VEGETABLE ASSOCIATION Food irradiation for the produce industry. Alexandria, 1986.11p.

URBAIN, W.M. Food irradiaton. London: Academic, 1986. p. 170-215.

VIEITES, R.L. Conservação pós-colheita do tomate através do uso da radiação gama, cera e saco de polietileno, armazenados em condições de refrigeração e ambiente. 1998. 131f. Tese (Livre-Docência) Faculdade de Ciências Agronômicas, Universidade Estadual Paulista, Botucatu, 1998.

ZHAO. M.; MOY. J.; PAULL, R. E. Effect of gamma-irradiation on ripening papaya pectin. Postharvest Biology and Technology, Amsterdan, v.8, p.209-22, 1996.

ZULETA, S. Technical and economic feasibility of irradiating mango and other fruits. Food irradiation Newsletter., Vienna, v. 2, n.13, p. 25-27, 1989. 